Tohoku J. exp. Med., 1975, 115, 47-52

\title{
Mycoplasma in Synovial Fluid from the Patients with Rheumatoid Arthritis
}

\author{
Michio Arai \\ Department of Orthopedic Surgery, Akita University School \\ of Medicine, Akita
}

\begin{abstract}
AraI, M. Mycoplasma in Synovial Fluid from the Patients with Rheumatoid Arthritis. Tohoku J. exp. Med., 1975, 115 (1), 47 52 — Mycoplasma colonies were found on culture of synovial fluid in 14 of 18 cases of rheumatoid arthritis. It was possible to demonstrate the colonies in primary eulture medium only by the method of hot water fixation and Giemsa staining. The organisms grown on solid medium were also observed electron-microscopically. _— mycoplasma; rheumatoid arthritis; synovial fluid
\end{abstract}

Recently some attention has been given to the hypothesis that mycoplasma is causally related to rheumatoid arthritis or rheumatic joint disease in man. Various culture techniques have been used to isolate mycoplasma from the joint fluid of rheumatoid individuals, but in most instances the results were negative except in the reports by Bartholomew (1965), Fahlberg et al. (1966), Williams (1968), Jansson et al. (197la, b, c) and Mårdh et al. (1973).

Our studies were undertaken in the hope that the primary isolation of mycoplasma from the joint fluid might be achieved by means of the culture technique of Mycoplasma pneumoniae, reported by Chanock et al. (1962).

\section{MeTHODS}

The medium consisted of Difco PPLO agar to which had been added $20 \%$ unheated horse serum, $10 \%$ fresh yeast extract and a concentration of 1:1,000 thalium acetate. Antibiotics were not employed for this investigation. A yeast extract was prepared by boiling $25 \mathrm{~g}$ of dried active yeast in $75 \mathrm{ml}$ of water for $15 \mathrm{~min}$. The boiled suspension was then centrifuged, and the supernatant was adjusted to $\mathrm{pH} 7.8$ with $1 \mathrm{~N}$ sodium hydroxide solution. The yeast extract and horse serum were added to the PPLO agar, after autoclaving and cooling to about $50^{\circ} \mathrm{C} .3 \mathrm{ml}$ of the final medium thus prepared were poured into sterile glass dishes of $4.5 \mathrm{~cm}$ diameter to prepare the agar plate. Synovial fluids from the knees of patients were obtained aseptically and 0.05 to $0.1 \mathrm{ml}$ of the fluid pipetted onto the agar surface, then spread to cover the total area of the agar plate. The plates, without sealing, were placed in a closed tin containing moist cottonwool and incubated aerobically at 36 to $37^{\circ} \mathrm{C}$. They were examined on the 4 th and 7 th days. Blocks of agar approximately $0.5 \mathrm{~cm}^{2}$ were cut from the plates. These agar blocks were placed face down on microscopic slides and fixed by the hot water fixation technique of Clark et al. (1961). After drying, the slides were immersed in methanol for 30 sec and then

Received for publication, October 28, 1974. 


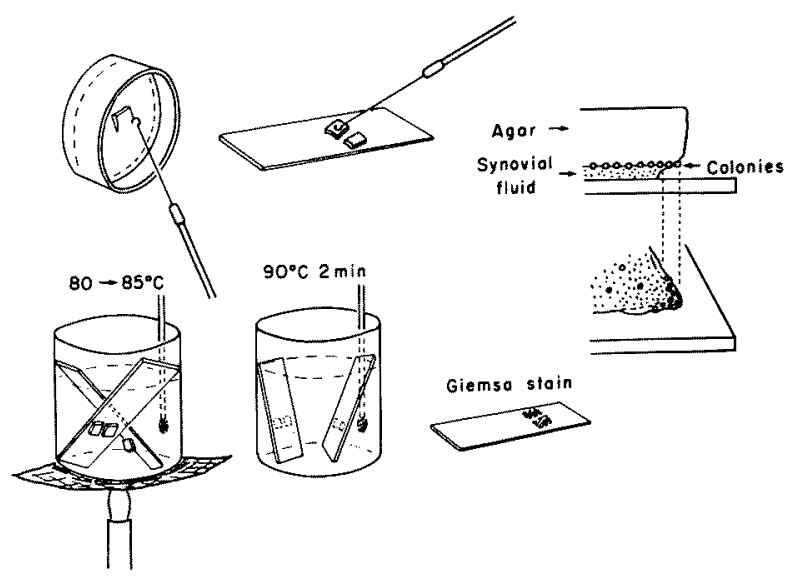

Fig. 1. Hot water fixation technique and adhesion of the colonies to the glass surface on the edges of the agar block.

stained with Giemsa stain for a period of 1 to $2 \mathrm{hr}$. Stock Giemsa stain was diluted 1:25 in $\mathrm{pH} 7.0$ phosphate buffer solution immediately before use.

\section{Results}

Photographs of typical colonies from 7 day cultures of mycoplasma are shown in Fig. 2. The majority of the colonies were observed to be in the size range of 5 to $15 \mu \mathrm{m}$, and the average size was about $10 \mu \mathrm{m}$. These colonies were characterized by their extremely minute size in comparison to the usually encountered mycoplasma colony type, e.g. colonies of $M$. pneumoniae (Fig. 3), and they resembled the "T-form" of Shepard (Shepard 1956) in shape and size.

Fig. 4 shows an electron micrograph of the same organism as in Fig. 2, grown on primary culture medium. This specimen was prepared from solid medium, by using the method of van Iterson and Ruys (1960).

Table 1 gives the cultural findings relating to 101 synovial fluids from 19 patients with polyarthritis. Mycoplasma colonies were found on culture of synovial fluid in 14 of 18 cases of rheumatoid arthritis. The patients were diagnosed according to the criteria of the American Rheumatism Association. In positive cases synovial fluid analyses revealed changes characteristic of rheumatoid arthritis in general; high polymorphonuclear cell count and positive latex fixation test. Moreover, by the staining procedure described by Shepard (1957), intracytoplasmic inclusions were demonstrated in synovial fluid cells (macrophages) from the rheumatoid patients in whom mycoplasma colonies were recovered. On the other hand, negative cultural results were obtained in the patients in the stage of remission. In one rheumatoid patient (Case 18) with non-inflammatory effusion which persisted 6 months after synovectomy, aspiration was performed 7 times repeatedly and all cultures were negative. In only 1 of 8 cases of chronic monoarthritis which could not be classified as rheumatoid arthritis was positive culture 


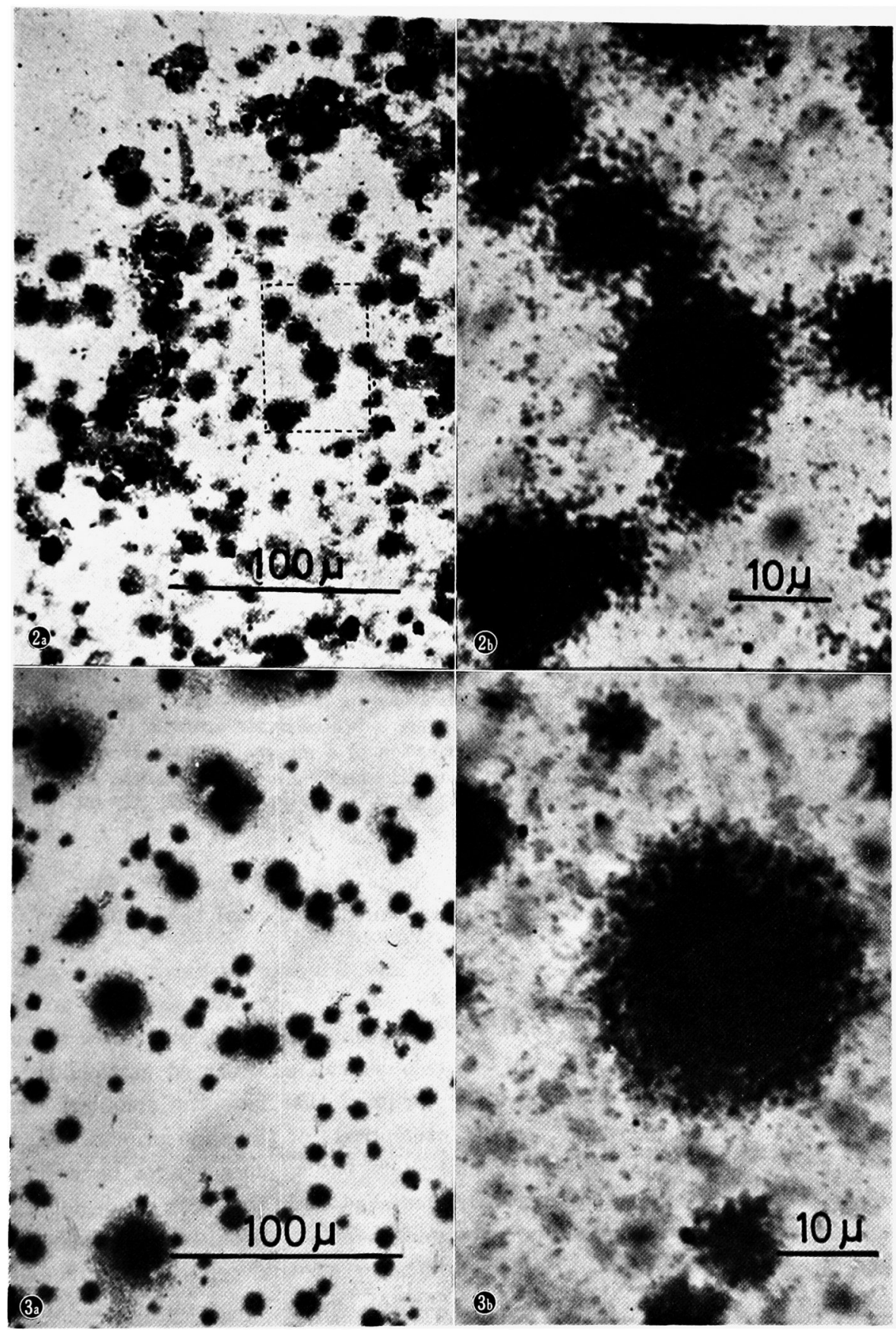

Fig. 2. Colonies from Case 2 (grown for 7 days on solid medium). Hot water fixation and Giemsa staining. a) Low magnification. Note the numerous round colonies and the debris of synovial fluid cells. b) High magnification. Note the pleomorphic organisms at the periphery of the colonies.

Fig. 3. Colonies of Mycoplasma pneumoniae ( $\mathrm{FH}$ strain). The staining is the same as in Fig. 2. Low (a) and high (b) magnification. 


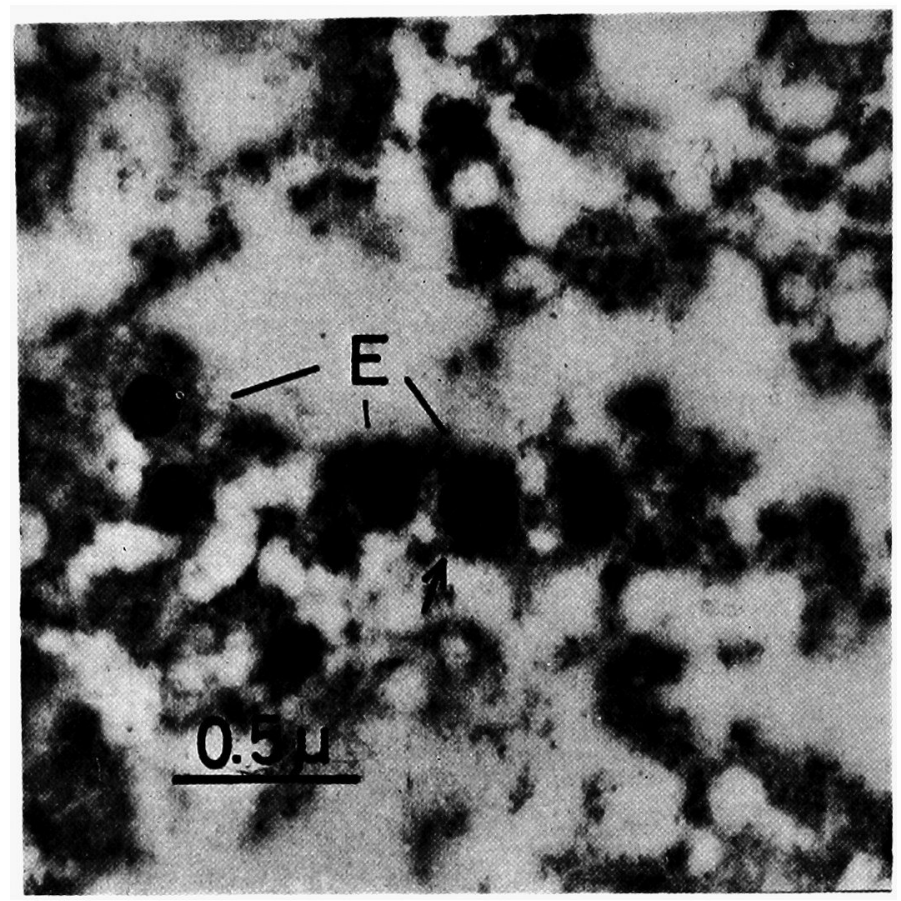

Fig. 4. Electron micrograph of the organisms grown on solid medium. The small dense cells are elementary bodies (E). The structure indicated by an arrow is considered to be an "assembly line" leading to the formation of a minimal reproductive unit. A large amount of autolyzed cells is also seen. The limiting membranes of the organisms consist of two dense layers and a lighter zone.

obtained. On the contrary, 36 synovial fluids from 33 patients with osteoarthritis and 4 cases of traumatic arthritis failed to reveal the colony by this method.

\section{Discussion}

Using the staining method numerous colonies can be observed particularly on the edges of the agar block. It can be assumed that here the colonies adhere closely to the glass surface (Fig. 1). In other parts of the slide preparation, the recognition of the colonies is very difficult, since the colonies cannot be adherent to the glass as a result of their development between the agar and deposited cellular materials of synovial fluid. Recognition of the colonies is not successful under direct agar microscopy, and identification of the colonies is frequently difficult by employing the staining procedure of Dienes. The failure of previous investigations to recover mycoplasma colonies in primary culture of synovial fluid from rheumatoid patients may possibly be explained by failure of the culture media to support growth of synovial fluid mycoplasma, or failure in recognition of colonies in primary culture. 
TABLE 1. Cultural results on synovial fluid from rheumatoid joints

\begin{tabular}{|c|c|c|c|c|c|c|}
\hline \multirow{2}{*}{\multicolumn{2}{|c|}{ Patient }} & \multirow{2}{*}{ Age Sex } & \multirow{2}{*}{ Diagnosis } & \multirow{2}{*}{ Latex test } & \multicolumn{2}{|c|}{ Number of fluids } \\
\hline & & & & & Cultured* & Positive \\
\hline 1 & T.K. & $36 \mathrm{M}$ & Classical RA & + & 18 & 10 \\
\hline 2 & M.Y. & $35 \mathrm{~F}$ & Classical RA & + & 13 & 8 \\
\hline 3 & T.H. & $38 \mathrm{~F}$ & Classical RA & + & 10 & 5 \\
\hline 4 & T.S. & $44 \mathrm{M}$ & Classical RA & + & 10 & 6 \\
\hline 5 & H.T. & $54 \mathrm{~F}$ & Classical RA & + & 6 & 5 \\
\hline 6 & T.S. & $31 \mathrm{M}$ & Classical RA & + & 8 & 4 \\
\hline 7 & S.T. & $48 \mathrm{~F}$ & Classical RA & + & 7 & 2 \\
\hline 8 & T.T. & $54 \mathrm{~F}$ & Classical RA & + & 5 & 2 \\
\hline 9 & H.S. & $62 \mathrm{~F}$ & Classical RA & + & 2 & 2 \\
\hline 10 & S.T. & $54 \mathrm{~F}$ & Classical RA & + & 2 & 1 \\
\hline 11 & H.M. & $25 \mathrm{~F}$ & Classical RA & + & 2 & 1 \\
\hline 12 & Y.K. & $64 \mathrm{~F}$ & Classical RA & + & 1 & 1 \\
\hline 13 & M.S. & $16 \mathrm{~F}$ & Classical RA & + & 2 & 1 \\
\hline 14 & K.A. & $17 \mathrm{M}$ & Classical RA & + & 3 & 1 \\
\hline 15 & Y.U. & $18 \mathrm{~F}$ & Classical RA & + & 2 & 0 \\
\hline 16 & T.I. & $24 \mathrm{~F}$ & Classical RA & + & 1 & 0 \\
\hline 17 & Y.O. & $21 \mathrm{~F}$ & Definite RA & \pm & 1 & 0 \\
\hline 18 & C.M. & $17 \mathrm{M}$ & Definite RA & - & $7^{\dagger}$ & 0 \\
\hline \multirow[t]{2}{*}{19} & Y.F. & $12 \mathrm{~F}$ & Rheumatic fever & - & 1 & 0 \\
\hline & Total & & & & 101 & $\overline{49}$ \\
\hline
\end{tabular}

* Different samplings from the patient.

$\dagger$ Synovial fluids after synovectomy.

RA: Rheumatoid arthritis.

From the present study, including controls from whom mycoplasmas were not isolated, it can be seen that the possibility of artifactual contamination would be eliminated. Therefore, these cultural findings might be taken to indicate that mycoplasmal infection is a more common event in rheumatoid arthritis than is generally appreciated. Unfortunately, however, the organism could not be successfully subcultured, and it was difficult to decide whether the organism was playing a pathogenic role. Details of its relationship to other strain were not studied in the present investigation.

\section{References}

1) Bartholomew, L.E. (1965) Isolation and characterization of Mycoplasma (PPLO) from patients with rheumatoid arthritis, systemic lupus erythematosus and Reiter's syndrome. Arthritis Rheum., 8, 376-388.

2) Chanock, R.M., Hayflick, L. \& Barile, M.F. (1962) Growth on artificial medium of an agent associated with atypical pneumonia and its identification as a PPLO. Proc. nat. Acad. Sci. USA, 48, 41-49.

3) Clark, H.W., Fowler, R.C. \& Brown, T.McP. (1961) Preparation of pleuropneumonialike organisms for microscopic study. J. Bact., 81, 500-502.

4) Fahlberg, W.J., Moor, R.W., Redmond, H.E. \& Brewer, E.J., Jr. (1966) Isolation of mycoplasma from human synovial fluids and tissues. Bact. Proc., 66, 48.

5) Iterson, W. van \& Ruys, A.Ch. (1960) The fine structure of the mycoplasmataceae (microorganisms of the pleuropenumonia group $=\mathrm{PPLO}$ ). 1. Mycoplasma hominis, $M$. 
fermentans and $M$. salivarium. J. Ultrastruct. Res., 3, 282-301.

6) Jansson, E., Mäkisara, P., Vainio, K., Snellman, O. \& Tuuri, S. (1971a) Further studies on mycoplasma in rheumatoid arthritis. Acta rheum. scand., 17, 227-235.

7) Jansson, E., Mäkisara, P., Vainio, K., Vainio, U., Snellman, O. \& Tuuri, S. (1971b) An 8-year study on mycoplasma in rheumatoid arthritis. Ann. rheum. Dis., 30, 506508 .

8) Jansson, E., Vainio, U., Snellman, O. \& Tuuri, S. (1971c) Search for mycoplasma in rheumatoid arthritis. Ann. rheum. Dis., 30. 413-418.

9) Mårdh, P. -A., Nilsson, F.J. \& Bjelle, A. (1973) Mycoplasmas and bacteria in synovial fluid from patients with arthritis. Ann. rheum. Dis., 32, 319-325.

10) Shepard, M.C. (1956) T-form colonies of pleuropneumonia-like organisms. J. Bact., 71, 362-369.

11) Shepard, M.C. (1957) Visualization and morphology of pleuropneumonia-like organisms in clinical material. $J$. Bact., 73, 162-171.

12) Williams, M.H. (1968) Recovery of mycoplasma from rheumatoid synovial fluid. In: Rheumatic Diseases, edited by J.J.R. Duthie \& W.R.M. Alexander, University Press, Edinburgh, p. 171. 